\title{
Simulating Performance of a Dual Angle Particle Monitor for Atmospheric Particulate Matter
}

Aerosol and Air Quality Research

\section{OPEN ACCESS}

Received: May 8, 2020

Revised: January 14, 2021

Accepted: January 15, 2021

${ }^{*}$ Corresponding Author: aranya@airvizinc.com

\section{Publisher:}

Taiwan Association for Aerosol Research

ISSN: $1680-8584$ print ISSN: 2071-1409 online

Copyright: The Author's institution. This is an open access article distributed under the terms of the Creative Commons Attribution License (CC BY 4.0), which permits unrestricted use, distribution, and reproduction in any medium, provided the original author and source are cited.

\author{
Charles D. Litton, Aranya Venkatesh", Jill Andersen, Dömötör Gulyás, \\ Sara Longo, Chris Bartley, Illah Nourbakhsh
}

Airviz Inc., Pittsburgh PA 15213, USA

\section{ABSTRACT}

Recent literature suggests that particle toxicity increases with decreasing particle diameter and increasing total particle surface area. Most inexpensive particle monitors are based upon light scattering and tend to lose sensitivity for particles with diameters less than about 0.3-0.35 $\mu \mathrm{m}$. This raises the question of whether the measurement of $\mathrm{PM}_{2.5}$ "misses" the potential impact of very small particles (e.g., below $0.3 \mu \mathrm{m}$ ) due to lack of sensitivity and/or the low mass concentrations that these particles contribute to the total $\mathrm{PM}_{2.5}$. On the other hand, measuring only ultrafine particles (e.g., below $0.1 \mu \mathrm{m}$ ) would exclude significant numbers of still very small particles. The focus of simulating a novel particle monitor in this study, is to address limitations in current inexpensive particle monitors, and to realize a particle monitor that may be more relevant to adverse health outcomes by measuring both $\mathrm{PM}_{0.3}$ and $\mathrm{PM}_{2.5}$. The monitor uses optical scattering techniques, measuring light scattering by the particles at two forward angles, to determine $\mathrm{PM}_{0.3}$ and $\mathrm{PM}_{2.5}$. Experimental data from particle monitor prototypes that were developed show good agreement with simulation results. Such a monitor, that is low-cost and easy to use, can provide information directly to the users so that they can be driven to action. In particular, low-income communities that are often impacted by poor air quality will be able to more affordably determine real-time ambient conditions and drive positive change by helping to identify pollution sources and appropriate mitigation measures.

Keywords: Particle monitor, Small particles, Dual angle

\section{INTRODUCTION}

While measurement of $\mathrm{PM}_{2.5}$ (particles smaller than $2.5 \mu \mathrm{m}$ ) is the recognized standard for fine particle air pollution, there is a growing body of evidence (Kelly and Fussell, 2012; Shi et al., 2016) to indicate that adverse health outcomes continue at levels less than half the average daily values of $12 \mu \mathrm{g} \mathrm{m}^{-3}$ specified by the Environmental Protection Agency (EPA) and considered to represent good to excellent air quality for PM 2.5. In 2005, Maynard and Kuempel (Maynard and Kuempel, 2005) concluded that particle toxicity increases with decreasing particle diameter and increasing total particle surface area, thus challenging mass-based risk evaluation approaches. These findings raise the question of whether or not the measurement of $\mathrm{PM}_{2.5}$ "misses" or "suppresses" the potential impact of very small particles (e.g., below $0.3 \mu \mathrm{m}$ ) due to lack of sensitivity and/or the low mass concentrations that these particles contribute to the total $\mathrm{PM}_{2.5}$.

Since deposition of particles within the alveolar regions of human lungs increases as the particle size decreases, it is reasonable to expect that the vast majority of PM-related adverse health problems may be due to the smaller particles contained within $\mathrm{PM}_{2.5}$. The Lung Deposited Surface Area (LDSA), has been proposed as a better metric to relate PM exposures to adverse health outcomes (Kuuluvainen et al., 2016) because it is the total particle surface that is in contact with lung tissue. Considerable attention has also been paid to measuring mass concentrations of ultrafine particles (PMo.1, particles with diameter less than $0.1 \mu \mathrm{m}$ ) and relating these concentrations to health problems (U.S. EPA, 2008; HEI, 2013). It is therefore of interest to determine if this 
measurement would be sufficient to ascribe adverse health outcomes at low $\mathrm{PM}_{2.5}$ concentrations or, perhaps, to determine if another metric might have greater validity that potentially could satisfy the constraints of both LDSA and ultrafine particle measurement.

There has been a recent surge in the market of inexpensive optical particle monitors over the past five years and to include all of them here is beyond the scope of this paper. However, most of these inexpensive particle monitors are based upon light scattering at a single forward angle of about $60^{\circ}$ and at typical wavelengths in the region of $780 \mathrm{~nm}$ to $880 \mathrm{~nm}$. These sensors typically differ primarily in the algorithms they use to convert optical signals to mass concentrations. Two types of currently available sensors, the Dylos (Unger, 1999) sensors and the Alphasense OPC-N2 sensors (Kaye and Hirst, 2010) differ from this approach by measuring the total light scattered from individual particles using novel optical systems to collect all of the scattered light from each particle as it passes through a well-defined scattering volume. But, regardless of the actual method used, Litton and Cauda (Litton and Cauda, 2019) found that typical monitors of either type begin to lose sensitivity when the average particle diameter of the scattering particles decreases below about $300 \mathrm{~nm}$ to $350 \mathrm{~nm}$. For instance, for the single angle scattering variety, the data shows that it takes 250 particles with a diameter of $90 \mathrm{~nm}$ to generate the same optical scattering intensity as one particle with a diameter of about $300 \mathrm{~nm}$ and for the sensors that measure total scattering, the results are even worse.

In a recent study related to potential health impacts by Geiss et al. (2016), it was concluded that over $99 \%$ of the LDSA from a multitude of different sources was due to particles with diameters less than $300 \mathrm{~nm}$. In addition, typical particle size distributions from some common combustion sources such as the exhaust of diesel engines (Hawley et al., 2014) or burning wood (Dietrich et al., 2015) indicate number mean particle diameters in the range of about $80 \mathrm{~nm}$ to $140 \mathrm{~nm}$ with typical geometric standard deviations of around 1.6 to 1.7. These studies indicate that measuring only ultrafine particles would exclude significant numbers of still very small particles, while measuring the contributions of particles with diameters less than $300 \mathrm{~nm}$ would include most, if not all, of these particles. In addition, optical sensors like the ones described above that become insensitive to these smaller particles could seriously underestimate the numbers, surface areas and masses of those smaller particles which may be the primary drivers of adverse health problems.

This background suggests that an improved particulate monitor that measured the mass and surface concentrations of both $\mathrm{PM}_{2.5}$ and $\mathrm{PM}_{0.3}$ could provide vital and relevant information on the effects of particulate matter on adverse health problems and that such a monitor would include both ultrafine particles and LDSA, two metrics that may have more significance in identifying health problems than only $\mathrm{PM}_{2.5}$. Inclusion of $\mathrm{PM}_{2.5}$ in a final device is important in that it then allows for reference to the existing standard for particulate mass concentrations as specified by the EPA and WHO.

Clearly, the use of optical scattering to measure particle concentrations, with current state-ofthe-art light sources and detectors, represents the least expensive approach and an approach that offers the best chance of success given that this technique can be applied in a strategic manner. Morawska et al. (2018) recently completed an extensive review of a number of commercial lowcost sensors air quality sensors and concluded that while these sensors perform well in the laboratory, they show poor response under natural conditions limited by issues such as lower ambient PM concentration compared to laboratory environments, and test aerosol composition that may differ significantly from ambient aerosols. In addition, these sensors are typically insensitive to particles below $350 \mathrm{~nm}$.

While the general need for an improved monitor for particulate matter that includes the tiniest of particles is presented above, there is also an environmental justice aspect to this development that cannot be ignored. Studies have shown that air pollution disproportionately affects lowincome and disadvantaged communities that often lack the resources to adequately address their problems. The availability of a particulate monitor such as the ones envisaged here, that are easy to use and interpret compared to expensive reference instruments that typically require more expertise, can provide information directly to the users so that they can be driven to action. In particular, low-income communities that are often impacted by poor air quality (Bell and Ebisu, 2012; Li et al., 2019; Yale-NUS College Singapore and Yale University's School of Forestry and Environmental Studies, 2019) will be able to more affordably determine real-time ambient 
conditions and drive positive change by helping to identify pollution sources, appropriate mitigation measures, and track the efficiency of these measures.

The focus of simulating a novel particle monitor in this study is to address limitations in current inexpensive particle monitors, and to realize a particle monitor that may be more relevant to adverse health outcomes by measuring both $\mathrm{PM}_{0.3}$ and $\mathrm{PM}_{2.5}$. The sections that follow describe the theory and potential implementation of one such dual angle particle monitor and simulate/measure its performance.

\section{METHODS}

\subsection{Theory}

For a monochromatic beam of light impinging upon particulate matter, the light is scattered in all directions producing three-dimensional angular intensity distributions. The shape of these angular intensity distributions and the actual intensities scattered at any given angle depend not only upon the monochromatic wavelength of the light source and the index of refraction of the scattering particles but also upon the shape and size of the particles. The influence of shape, or morphology, is most evident when the particles are dust particles, or other mechanically generated particles, or particles that form fractal-like aggregates with shapes ranging from long, irregular chains of primary particles to more densely packed, or clumped primary particles. These fractallike particles are most frequently observed from combustion sources, but even for these aggregates, the data suggests that the normalized scattering cross-sections are those of the smaller primary particles comprising the aggregate typically amplified by the number of primary particles per aggregate so that the angular intensities still scale with the smaller, primary particles. Further, the intensity ratio at two different angles is independent of the number of primary particles and depends only upon the normalized scattering cross-sections for the primary particles at each angle. While the actual shape of the particles will have some influence on the angular intensities, it is beyond the scope of this paper to discuss this aspect of the problem in any detail. The dual angle particle monitor described below operates by taking advantage of the differences in angular intensity distributions as a function of particle diameter through the use of a dimensionless intensity ratio that serves as a surrogate for particle size, where the particles are idealized as spheres in order to simplify the analyses.

In the device, the light source used is a simple diode laser operating at a wavelength of $\lambda=$ $650 \mathrm{~nm}$. In this device, one photodiode is positioned to measure the intensity scattered at a forward angle of $30^{\circ}$, while a second photodiode is positioned to measure the intensity scattered at the forward angle of $60^{\circ}$. The normalized angular scattering efficiency at either angle, $I_{\theta}$, computed using the simple Barber and Hill Mie scattering algorithm (Barber and Hill, 1990) for scattering from a sphere, is measured by each photodiode and the corresponding photodiode current converted to a voltage, $\Delta V_{\theta}$. For discrete particle diameters, $d(\mathrm{~cm})$, the intensity scattered at each angle, $\theta$, is measured and converted to a voltage, $\Delta V_{\theta}$, using the following expression

$\Delta V_{\theta}=\left\{(\pi / 4) \cdot d^{2} \cdot P_{S} \cdot d \Omega \cdot R_{D} \cdot G_{A} \cdot V_{S C A T}(\theta) \cdot N\right\} I_{\theta}$

where

$P_{S}$ is the diode laser flux $\left(\mathrm{W} \mathrm{cm}^{-2}\right)$;

$d \Omega$ is the solid angle subtended by the detector (sr);

$R_{D}$ is detector responsivity $(\mathrm{A} / \mathrm{W})$ at the diode laser wavelength of $650 \mathrm{~nm}$;

$G_{A}$ is the current to voltage amplification $\left(5 \times 10^{8} \mathrm{~V} / \mathrm{A}\right)$;

$V_{\text {SCAT }}(\theta)$ is the scattering volume at the angle $\theta,\left(6.0 \times 10^{-3} / \sin \theta\right)$;

$N$ is the number of particles per $\mathrm{cm}^{3}$.

Eq. (1) can be divided into two components - one component containing only those parameters that relate to the particles being measured and the second component containing only those parameters that relate to the device configuration and supporting electronics. If this second component is denoted by the parameter, $\beta$, then Eq. (1) can be rewritten as

$\Delta V_{\theta}=\left\{(\pi / 4) \cdot d^{2} \cdot N \cdot I_{\theta}\right\} \cdot \beta$ 
where, $\beta=P_{S} \cdot d \Omega \cdot R_{D} \cdot G_{A} \cdot V_{S C A T}(\theta)$ and is defined as the instrument function.

Eqs. (1) and Eq. (2) apply strictly to particles with discrete diameters and measured particle number concentrations, $N$, at those diameters. It is often the case that such discrete data is not readily available and the particle diameters described in terms of some distribution function. $A$ particle size distribution commonly used to describe atmospheric particles is the lognormal distribution, defined by the geometric number mean diameter, $d_{G}$, and geometric standard deviation, $\sigma_{G}$. Using the lognormal distribution, the diameter of average surface, $d_{20}$, and diameter of average mass, $d_{30}$, are then defined in terms of $d_{G}$ and $\sigma_{G}$, by the following expressions:

$d_{20}=d_{G} \cdot \exp \left\{\left[\ln \left(\sigma_{G}\right)\right]^{2}\right\}$

$d_{30}=d_{G} \cdot \exp \left\{\left[1.5 \ln \left(\sigma_{G}\right)\right]^{2}\right\}$

When $N$ is the number of particles per $\mathrm{cm}^{3}$, the diameters are in $\mathrm{cm}$, and the density, $\rho$, is assumed to be $1.0 \mathrm{~g} \mathrm{~cm}^{-3}$, the mass concentration, $M$, in $\mathrm{mg} \mathrm{m}^{-3}$, is given by

$M=1 \times 10^{9}(\pi / 6)\left(d_{30}\right)^{3} \mathrm{~N}$

For lognormal distributions Eq. (2) can be rewritten as

$\Delta V_{\theta}=\left\{(\pi / 4) \cdot\left(d_{20}\right)^{2} \cdot N \cdot I_{\theta}\right\} \cdot \beta$

Eqs. (3)-(6) can be combined to yield an equivalent expression for mass sensitivity in units of volts per $\mu \mathrm{g} \mathrm{\textrm {m } ^ { - 3 }}$

$\Delta V_{\theta} / M=1.5 \times 10^{-9}\left[\beta\left\{\exp \left[-2.5\left[\ln \left(\sigma_{G}\right)\right]^{2}\right\} / d_{G}\right] I_{\theta}\right.$

Eq. (7) is a useful expression when the mass concentration of particles is known and the size distribution is either known or can be approximated using a lognormal distribution.

In order to use the devices, it is necessary to perform calibration experiments by exposing them to particles with a known particle size distribution and a known mass or total number concentration. To perform such calibrations dilute mixtures of dioctyl phthalate (DOP) in ethanol were atomized to produce very narrow polydispersions. The particles generated were subsequently measured using a TSI NANO SMPS (for particle diameters < $350 \mathrm{~nm}$ ) and a TSI Optical Particle Sizer (OPS) for particles in the size range $350 \mathrm{~nm}<d<10000 \mathrm{~nm}$. A photograph of the experimental set-up is shown in Fig. 1.

\subsection{Laboratory Testing}

Before conducting a series of experiments, the lab room was prepped by dusting off equipment and running a room air purifier for 24 hours. The sensors were then placed into a polymethyl methacrylate (PMMA) sample box and allowed to acclimate to box conditions for a minimum of 30 minutes prior to testing. Temperature and humidity values were recorded from the sample box during each experiment. The OPS and SMPS, used to collect the spectra of particles and calculate particle concentration levels, were pre-warmed for 30 minutes prior to testing, and sensor baselines were typically recorded for $15 \mathrm{~min}$ prior to introducing aerosols into the sample chamber.

Tests were performed DOP (dioctyl phthalate, density of $0.978 \mathrm{~g} \mathrm{~cm}^{-3}$ at $20^{\circ} \mathrm{C}$ and a refractive index of 1.485) (National Center for Biotechnology Information, 2020) generated from a TSI Model 3076 atomizer using dilute solutions of DOP in ethanol at various mixture ratios to obtain different particle sizes. When testing with DOP particles it was necessary to fill the dilution sample bag with filtered house air and then add in particles for $30 \mathrm{~s}$ and allow them to mix thoroughly before flowing them into the PMMA sample box with sensors. The PMMA sample box was also pretreated with antistatic spray prior to running an experiment.

The NANO SMPS Model 3910 has 13 discrete size bins with diameter midpoints beginning at $11.5 \mathrm{~nm}$ and ending with $365.2 \mathrm{~nm}$. During a typical experiment, the number of particles per $\mathrm{cm}^{3}$ are counted and placed in the appropriate size bin so that either a number, surface, or mass size distribution can be obtained, as well as total number, surface, and mass. For the OPS, 15 size bins 


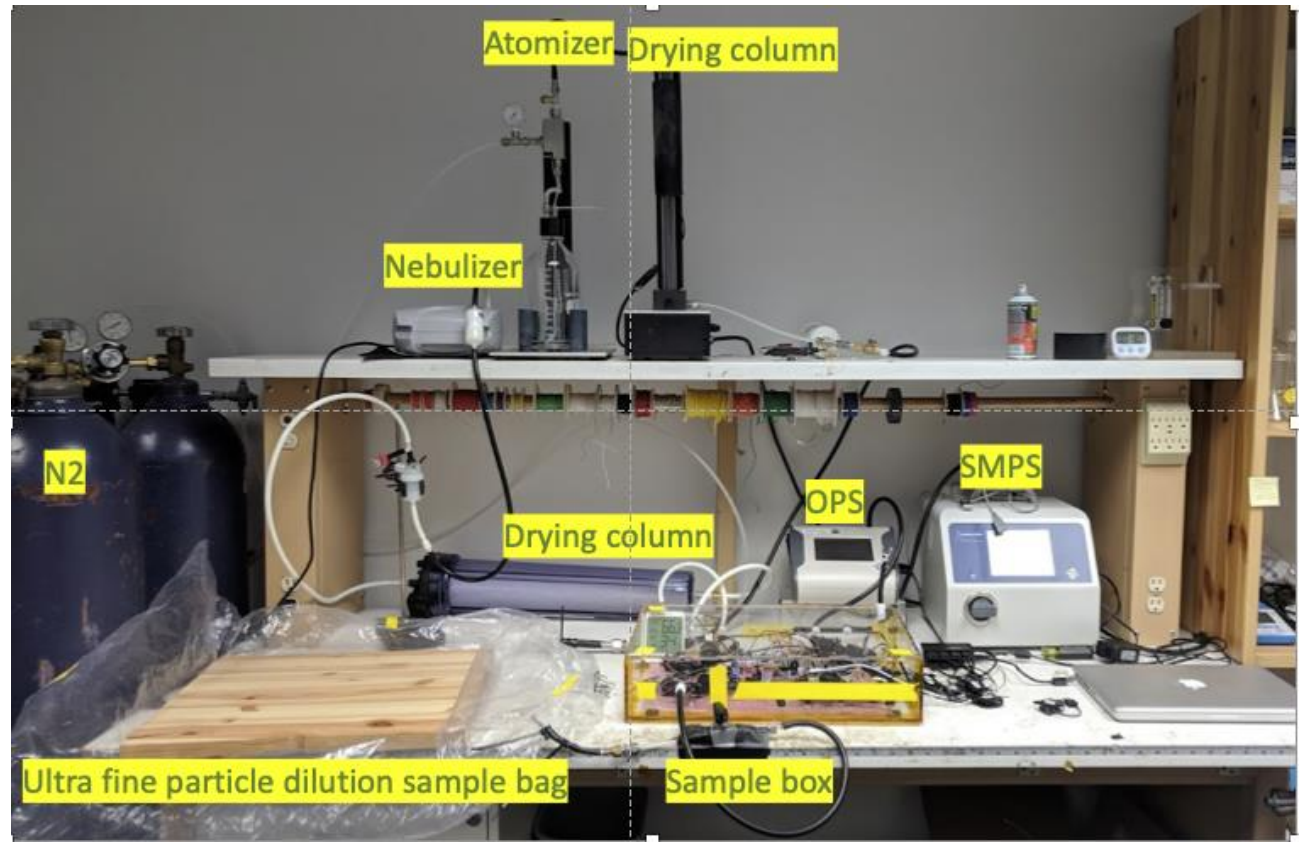

Fig. 1. Photograph of the experimental arrangement used to calibrate and evaluate prototype dual angle sensors.

with diameter midpoints ranging from $374 \mathrm{~nm}$ to $8031 \mathrm{~nm}$ are filled in a similar manner to allow for similar particle size distribution estimates to be made. When the generated particles consist only of particles with diameters less than $350 \mathrm{~nm}$ or, alternatively, only of particles with diameters greater than $350 \mathrm{~nm}$, then either the NANO SMPS data or the OPS data, respectively, can be used to determine the device constant, $\beta$, from the following expression

$\Delta V_{\theta}=(\pi / 4) \cdot \beta \cdot 10^{-14} \cdot \sum d_{i}^{2} \cdot N_{i} \cdot\left(I_{\theta}\right)$

where the subscript " $i$ " refers to a specific bin with midpoint, $d_{i}(\mathrm{~nm})$, containing $N_{i}$ particles per $\mathrm{cm}^{3}$ and the factor of $10^{-14}$ is used to convert the diameter in $\mathrm{nm}$ squared to diameter in $\mathrm{cm}$ squared.

For the data discussed in Table 1, four units of the same sensor model were placed into the sample chamber box with inlets facing towards the center of the sample box and a few inches away from the aerosol instrumentation sampling outlets (see Fig. 1) and the sensors allowed to acclimate to the box conditions for 30 minutes prior to testing. For this experiment DOP was diluted with ethanol (EtOH) to $0.3 \% \mathrm{v} / \mathrm{v}$ to generate particles with an approximate number mean diameter of $150 \mathrm{~nm}$ (the actual measured average value was $165.9 \mathrm{~nm}$ with a geometric standard deviation (GSD) of 1.65). The solution was aerosolized with the TSI atomizer and subsequently dried with a column heater to evaporate off the ethanol. The generated particles were then introduced into the dilution sample bag along with filtered house air for $30 \mathrm{~s}$ and mixed thoroughly before flowing into the PMMA sample box at a rate of 2.2 LPM via applied constant positive pressure to the bag. Table 1 displays the average NANO SMPS data from eleven (11) consecutive 60 second scans obtained during this experiment when the measured particle concentrations had reached their peak and values had stabilized. The average sums, $\sum d_{i}^{2} \cdot N_{i} \cdot(/ 30)_{i}$ and $\sum d_{i}{ }^{2} \cdot N_{i} \cdot\left(I_{60}\right)_{i}$, from this table were calculated to be $1.242 \times 10^{8}$ and $6.091 \times 10^{7}$, respectively and are reported at the bottom of the table. The voltages $V_{30}=0.0339 \mathrm{~V}$ and $\mathrm{V}_{60}=.01096 \mathrm{~V}$, also reported at the bottom of the table, are average values measured by the four prototype devices averaged over the same time period of the eleven NANO SMPS scans.

Using Eq. (8) above and the respective sums and voltages quoted above, for $\theta=30^{\circ}$, a value of $\beta_{30}=3.48 \times 10^{4}$ is obtained and for $\theta=60^{\circ}$, a value of $\beta_{60}=2.29 \times 10^{4}$ is obtained. The lower value of $\beta_{60}$ is due primarily to the smaller scattering volume and may also reflect slight differences in the laser diode energy flux compared to the flux at the $30^{\circ}$ scattering angle. Consequently, by 
Table 1. Average data from eleven consecutive NANO SMPS scans showing the number of particles per $\mathrm{cm}^{3}$ measured in each of the 13 size bins.

\begin{tabular}{|c|c|c|c|c|c|}
\hline$d_{i}(n m)$ & $\mathrm{N}_{\mathrm{i}}\left(\mathrm{p} \mathrm{cm}^{-3}\right)$ & $\left(I_{30}\right)_{i}\left(s r^{-1}\right)$ & $\left(I_{60}\right)_{i}\left(s r^{-1}\right)$ & $\left(d_{i}\right)^{2} N_{i}\left(I_{30}\right)_{i}$ & $\left(d_{i}\right)^{2} N_{i}\left(I_{60}\right)_{i}$ \\
\hline 11.5 & $1.77 \mathrm{E}+02$ & $1.10 \mathrm{E}-07$ & $7.83 \mathrm{E}-08$ & $2.56 \mathrm{E}-03$ & $1.83 \mathrm{E}-03$ \\
\hline 15.4 & $3.90 \mathrm{E}+00$ & $3.53 \mathrm{E}-07$ & $2.52 \mathrm{E}-07$ & $3.26 \mathrm{E}-04$ & 2.33E-04 \\
\hline 20.5 & $1.32 \mathrm{E}+02$ & $1.11 \mathrm{E}-06$ & 7.93E-07 & $6.16 \mathrm{E}-02$ & $4.40 \mathrm{E}-02$ \\
\hline 27.4 & $1.01 \mathrm{E}+03$ & $3.56 \mathrm{E}-06$ & $2.54 \mathrm{E}-06$ & $2.69 \mathrm{E}+00$ & $1.92 \mathrm{E}+00$ \\
\hline 36.5 & 4.77E+02 & $1.13 \mathrm{E}-05$ & $8.02 \mathrm{E}-06$ & $7.17 \mathrm{E}+00$ & $5.10 \mathrm{E}+00$ \\
\hline 48.7 & $4.78 \mathrm{E}+01$ & $3.62 \mathrm{E}-05$ & $2.56 \mathrm{E}-05$ & $4.10 \mathrm{E}+00$ & $2.91 \mathrm{E}+00$ \\
\hline 64.9 & $3.78 \mathrm{E}+02$ & $1.16 \mathrm{E}-04$ & $8.18 \mathrm{E}-05$ & $1.85 \mathrm{E}+02$ & $1.30 \mathrm{E}+02$ \\
\hline 86.6 & 4.07E+03 & $3.81 \mathrm{E}-04$ & $2.65 \mathrm{E}-04$ & $1.16 \mathrm{E}+04$ & $8.08 \mathrm{E}+03$ \\
\hline 115.5 & $1.02 \mathrm{E}+04$ & $1.27 \mathrm{E}-03$ & $8.66 \mathrm{E}-04$ & $1.72 \mathrm{E}+05$ & $1.18 \mathrm{E}+05$ \\
\hline 154 & $1.49 \mathrm{E}+04$ & 4.33E-03 & $2.85 \mathrm{E}-03$ & $1.54 \mathrm{E}+06$ & $1.01 \mathrm{E}+06$ \\
\hline 205.4 & $1.51 E+04$ & $1.49 \mathrm{E}-02$ & $9.24 \mathrm{E}-03$ & $9.53 E+06$ & $5.90 E+06$ \\
\hline 273.8 & $1.05 E+04$ & 4.95E-02 & $2.71 \mathrm{E}-02$ & $3.91 E+07$ & $2.14 \mathrm{E}+07$ \\
\hline 365.2 & $3.33 \mathrm{E}+03$ & $1.66 \mathrm{E}-01$ & 7.31E-02 & $7.39 \mathrm{E}+07$ & $3.24 \mathrm{E}+07$ \\
\hline \multirow[t]{2}{*}{ Total N } & $6.04 \mathrm{E}+04$ & Average & Sums: & $1.24 \mathrm{E}+08$ & $6.09 \mathrm{E}+07$ \\
\hline & & Average & Voltages: & $V_{30}=3.39 E-02$ & $V_{60}=1.10 E-02$ \\
\hline
\end{tabular}

measuring the device voltage outputs, $\mathrm{V}_{30}$ and $\mathrm{V}_{60}$, corresponding to individual scans of the NANO SMPS and performing similar computations, device constants can be obtained for each scan. The values above are for 11 consecutive scans (11 minutes) and represent the average number concentrations in each particle size bin, the average sensor voltages (for four sensors) over the same time interval and, therefore, the average values of these constants. The standard deviation for the constants derived from this set of data, expressed as a percentage of the average values, is $\pm 7.3 \%$. For the average voltages in Table $1, V_{30}$ and $V_{60}$, the standard deviations for the four sensors used, expressed as a percentage of the average voltages, were $\pm 2.9 \%$ and $\pm 0.50 \%$, respectively, indicating a high degree of uniformity between the four sensors.

When the particle size distribution is defined by a continuous lognormal distribution (see Eq. (7)), it is found that the calculated values of $\beta$ increase. For instance, in the above example, using the average distribution over the same time interval, defined by $d_{G}=165.9 \mathrm{~nm}$ and $\sigma_{G}=$ 1.65 , the calculated values for $I_{30}$ and $I_{60}$ are, respectively, 0.03471 and 0.01392 . At an average mass concentration of $305.1 \mu \mathrm{g} \mathrm{m}^{-3}$ and average voltages of $V_{30}=33.9 \mathrm{mv}$ and $V_{60}=10.96 \mathrm{mv}$, $V_{30} / M=0.1111 \mathrm{mV} /\left(\mu \mathrm{g} \mathrm{m}^{-3}\right)$ and $V_{60} / M=0.0359 \mathrm{mv} /\left(\mu \mathrm{g} \mathrm{m}^{-3}\right)$, resulting in constants of $\beta_{30}=5.40$ $\times 10^{4}$ and $\beta_{60}=5.34 \times 10^{4}$. When discrete particle size data are not available and size distributions are used to approximate the sizes, then the latter values should be used.

Fig. 2 shows a heat map of the particle mass distribution obtained from the reference instruments for the set of data discussed above. It is worth noting that, on a mass basis, the average mass-weighted diameters always shift to larger diameters compared to geometric number mean diameters. So, for these data the greatest mass concentration appears to be from particles with diameters of around $300 \mathrm{~nm}$, in excellent agreement with a diameter of average mass of $309 \mathrm{~nm}$ calculated from the lognormal distribution measured for this experiment $\left(d_{G}=\right.$ $165.9 \mathrm{~nm}$ and $\left.\sigma_{G}=1.65\right)$.

\subsection{Materials}

DOP (dioctyl phthalate) was purchased from Sigma Aldrich (St. Louis, MO). Ethyl Alcohol (99\%) was purchased from PHARMCO_AAPER Absolute, Anhydrous 200 Proof.

\section{RESULTS AND DISCUSSION}

\subsection{Running the Simulations}

It is not the intent of this paper to describe detailed calibration data but rather to present the 


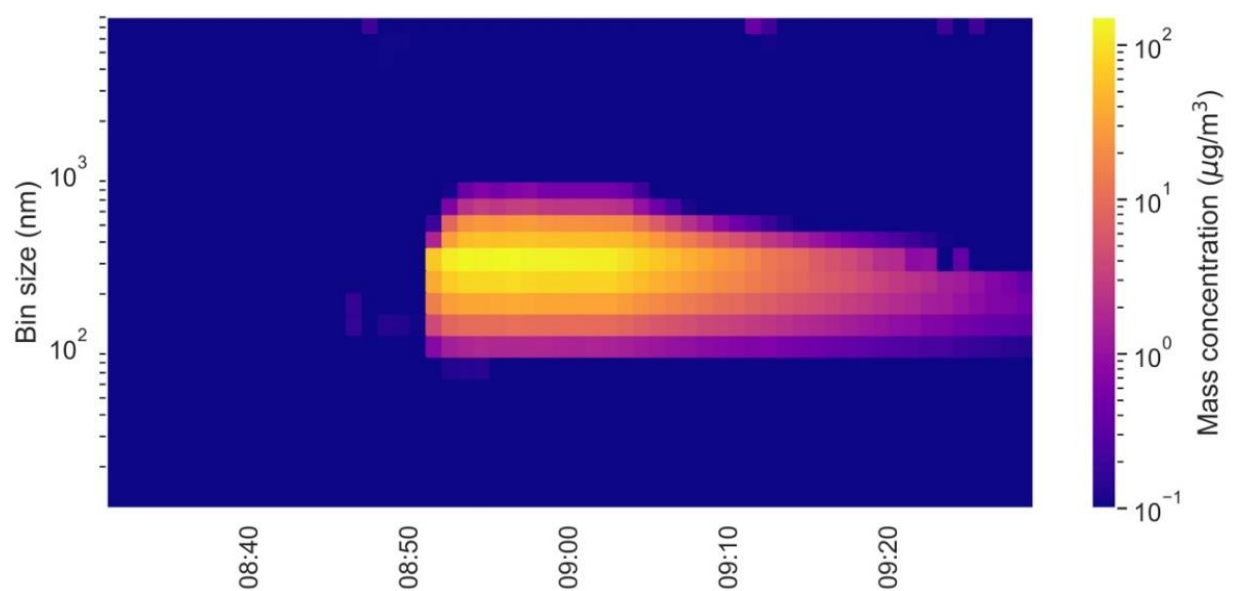

Fig. 2. A heat map of the mass distribution of particle diameters measured for the data discussed previously

results of the calibration data and then to apply those results to simulate the device response to actual particle sizes and distributions measured. Once these simulated voltage responses are calculated, it is instructive to define the calculated responses per unit mass concentration as a function of the dimensionless ratio, $V_{30} / V_{60}$. This ratio represents a parameter which depends upon the average sizes of the particles and can be used, ultimately, to provide a better determination of particulate mass concentrations and the fraction of the total mass concentration that is due to particles with diameters less than $300 \mathrm{~nm}$. Discussion of this aspect of the device will be saved for a later section.

When we initially explored this technique as a simple method to effect some degree of particle size discrimination, angular intensities were calculated for mixtures of two idealized size distributions and it was shown that the fraction of very tiny particles (which we defined as all particles with diameters less than $300 \mathrm{~nm}$ or $\mathrm{PM}_{0.30}$ ) present in the total mass of $\mathrm{PM}_{2.5}$ particles could be expressed as a function of angular intensity ratios. In follow-up work, laboratory data was used to verify that measured angular intensity ratios were in excellent agreement with theoretically calculated intensity ratios when measured for very narrowly distributed polydisperse particles with diameters ranging from about $100 \mathrm{~nm}$ to about $800 \mathrm{~nm}$. In the real world, these ideal mixtures and ideal size distributions are unlikely to occur so the question becomes as to how this concept might apply to the measurement of particles actually present in the atmosphere. But before looking at this in greater detail as it applies to real-world particulate matter, it is instructive to apply this simulation to laboratory data to assess the validity of the approach.

To do this, the dual angle sensors were exposed to particles of known composition dioctyl phthalate (DOP), with an index of refraction equal to 1.486 and the size distributions measured continuously at one-minute intervals using a NANO SMPS with 13 different size bins. For each of the one-minute distributions, Equation 8 was applied using the constants of $\beta_{30}=3.48 \times 10^{4}$ and $\beta_{60}=2.29 \times 10^{4}$ and the calculated Mie cross-sections for each particle diameter bin and the sum over all diameter bins calculated to generate the expected voltages, $V_{30}$, and $V_{60}$. The results of these simulations are compared to the measured voltages, $V_{30}$ and $V_{60}$, in Fig. 3.

From this figure, it is obvious that these simulations yield predicted output voltages at the two angles that are in good to excellent agreement with the simultaneously measured values. This type of agreement indicates that the approach can be applied to real-world atmospheric particulate matter when there exist good particle size distribution data, such as the data generated from SMPS instruments, or similar, where number concentrations are obtained at discrete particle diameters. The following sections describe such data and the subsequent analyses.

\subsection{Simulations Using Ambient Particle Size Distributions}

The United Kingdom's Department for Environment Food \& Rural Affairs (DEFRA) maintains certain monitoring sites in London and other parts of the UK where Scanning Mobility Particle Size (SMPS) instruments measure ambient particle size distributions in 50 discrete diameter bins 

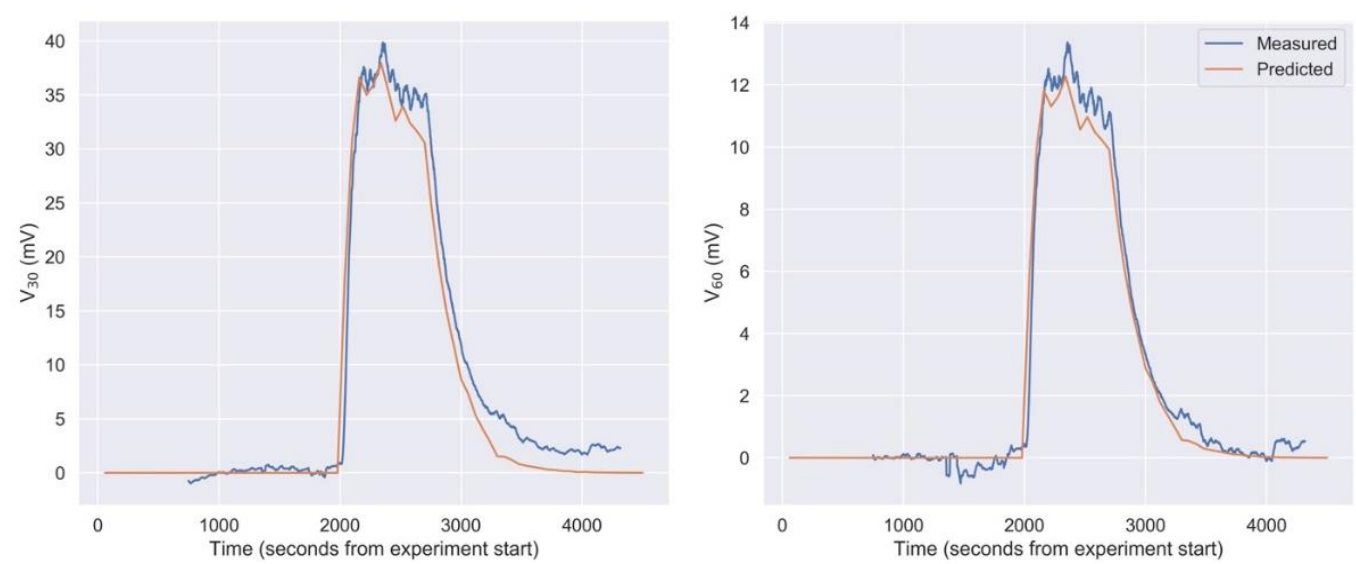

Fig. 3. Comparison of the measured voltage, $V_{30}$ (left panel) and $V_{60}$ (right panel) to the value predicted from the simulation using NANO SMPS discrete particle size data.

from $16.55 \mathrm{~nm}$ to $604.3 \mathrm{~nm}$ every 15 minutes 365 days a year (DEFRA, 2019). These data date back to 1997 and represent a unique repository of detailed atmospheric particle size and concentration data that has been validated by UK scientists to eliminate any bad data due to instrument problems or other potential sources of error that could lead to erroneous data before being posted on the DEFRA website. In 2017 we obtained access to these data and began to conduct simulations of the manner in which a proposed optical sensor might respond to real-life particulate matter. In some of these initial simulations approximate sensor response functions were used just to assess how a sensor might typically respond to scattering signals at one or more forward scattering angles. The results of these simulations were extremely encouraging.

In order to execute more realistic simulations, it was necessary to determine the instrument response parameters, $\beta_{30}$ and $\beta_{60}$, as discussed above and to calculate normalized, differential Mie scattering cross-sections for each of the particle diameters comprising the 50 particle size bins of the SMPS and at some representative values of the index of refraction. Data on refractive indices of atmospheric aerosols (Dick et al., 2007) that tend to bracket the range of possible values indicated a value of the index of refraction of $m=1.46$ for organic aerosols and an index of refraction typical of black carbon particles of $m=1.93-0.66 \mathrm{i}$.

Because there is significant uncertainty in the type of particle being measured, an average value for the index of refraction from these two values, $m=1.695-0.33 i$, was used in the calculations to demonstrate the concept and its potential for more general application. The resultant calculated efficiencies for such particles are shown in Fig. 4, above, for the two angles used in these simulations, $30^{\circ}$ and 60 . While it is expected that the index of refraction encountered in any actual end-use configuration may be different from the value used here, these results can be modified if needed by using a more accurate refractive index if it is known.

To calculate the voltages expected from the dual angle device these normalized Mie cross sections for each bin are multiplied by the bin number concentration measured for the given particle size and the square of the corresponding bin particle diameter. The sum over all bins is then multiplied by the respective constants, $\beta_{30}=3.48 \times 10^{4}$ and $\beta_{60}=2.29 \times 10^{4}$ to yield simulated voltages, $V_{30}$ and $V_{60}$, for that particle size distribution (see Equation 8 ). In a similar manner, the total mass concentration was calculated from the sum of the product of the cube of the diameter and the number concentration for each size bin. To determine the fraction of this total mass concentration that is due to very tiny $(d<300 \mathrm{~nm})$ particles, the sum of these mass bins up to the diameter of $300 \mathrm{~nm}$ is divided by the total sum to yield the mass fraction of these tiny particles (PM0.30). In addition, the calculated voltage at each scattering angle is divided by the total mass concentration to yield the sensitivity in terms of volts per unit mass concentration. Similar analyses were also done for total surface area and very tiny particle surface fraction should the need exist to use a different, and perhaps more relevant, metric than mass concentration.

To date, at least 22 data sets have been analyzed using this technique, representing a total of about 19 years' worth of data from four different monitoring sites. For each monitoring site, we have determined the average mass sensitivities, $V_{30} / M$ and $V_{60} / M$, the average ultrafine mass 


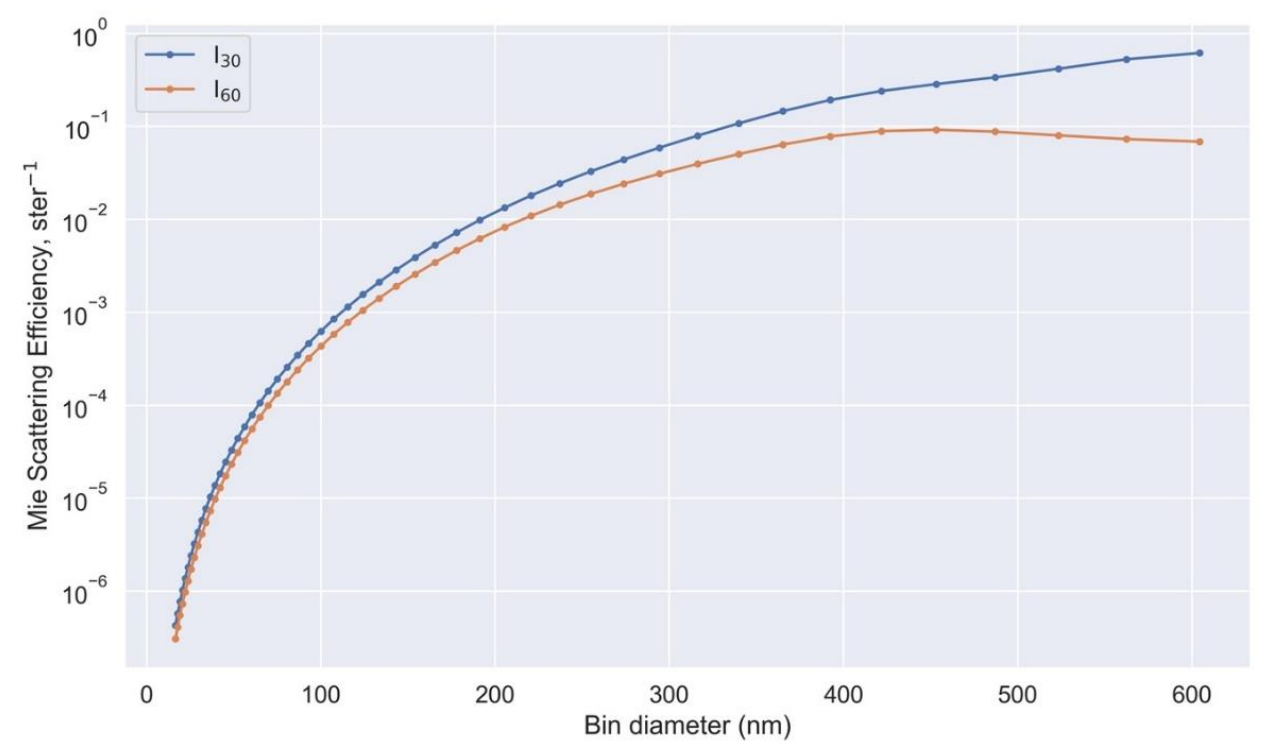

Fig. 4. Normalized Mie scattering cross sections for the 50 particle size bins of the SMPS at forward scattering angles of $30^{\circ}$ and $60^{\circ}$ for aerosols with $m=1.695-0.33$ i.

fraction, $\mathrm{M}_{0.30} / \mathrm{M}_{\text {Tот, }}$, the average ultrafine surface fraction, $\mathrm{S}_{0.30} / \mathrm{S}_{\text {Tот, }}$, and the average mass weighted diameter as functions of the angular intensity ratio. In these calculations, an average value of the refractive index, $m=1.695-0.33 i$, was used as some average for $50 / 50$ combinations of organic and black carbon particles. The mass sensitivities, $V_{30} / M$ and $V_{60} / M$, are shown, respectively, in Figs. 5 and 6 . The mass fraction and the surface fraction of PM0.30 are shown, respectively, in Figs. 7 and 8, while the mass weighted average diameter is shown in Fig. 9. Similarly, the surface sensitivities, $V_{30} / S$ and $V_{60} / S$, are shown, respectively, in Figs. 10 and 11 .

Figs. 5-7 provide the information necessary to determine total mass concentrations (assumed to be $\mathrm{PM}_{2.5}$ ) and the mass fraction of the total mass due to particles with diameters less than $300 \mathrm{~nm}$, or $\mathrm{PM}_{0.30}$. In operation, the sensor would read the voltages, $\mathrm{V}_{30}$ and $\mathrm{V}_{60}$, subtract the baselines (zero particle values) and then form the resultant ratio. The ratio would be used to determine the mass sensitivities, $V_{30} / M$ and $V_{60} / M$, for the measured ratio (Figs. 5 and 6 respectively). The total $\mathrm{PM}_{2.5}$ mass would then be determined by dividing measured voltage differences above background by the corresponding sensitivities and then taking the average value of the two calculated mass concentrations.

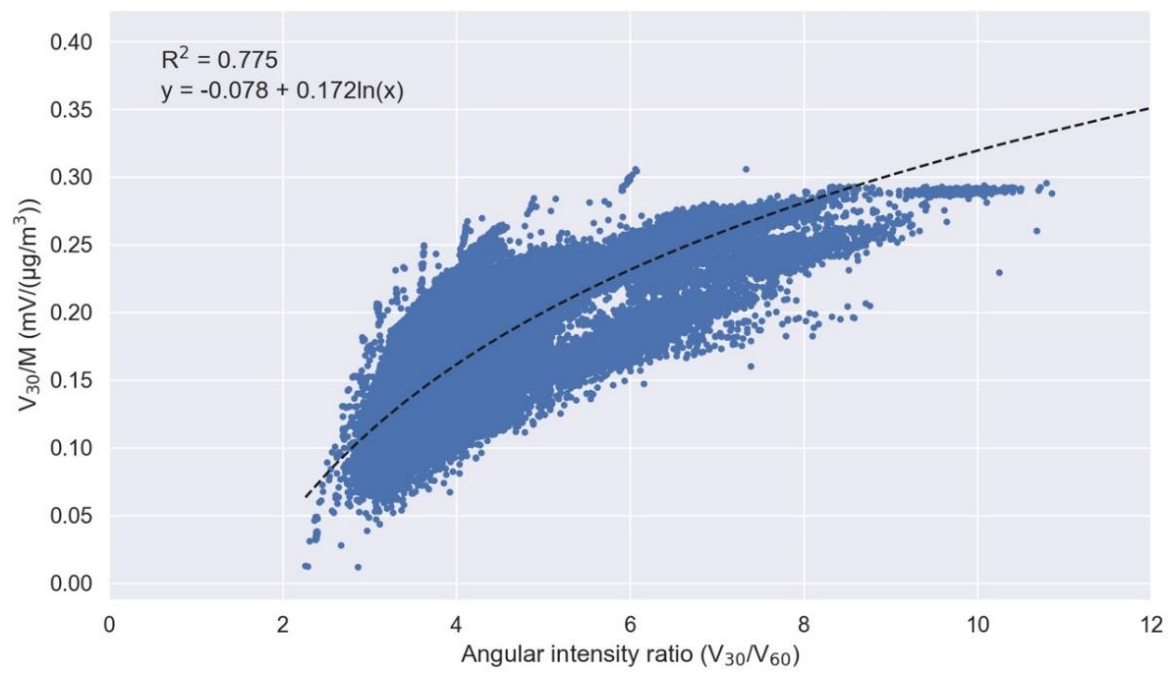

Fig. 5. Average mass sensitivity at $30^{\circ}$ as a function of the angular intensity ratio. 


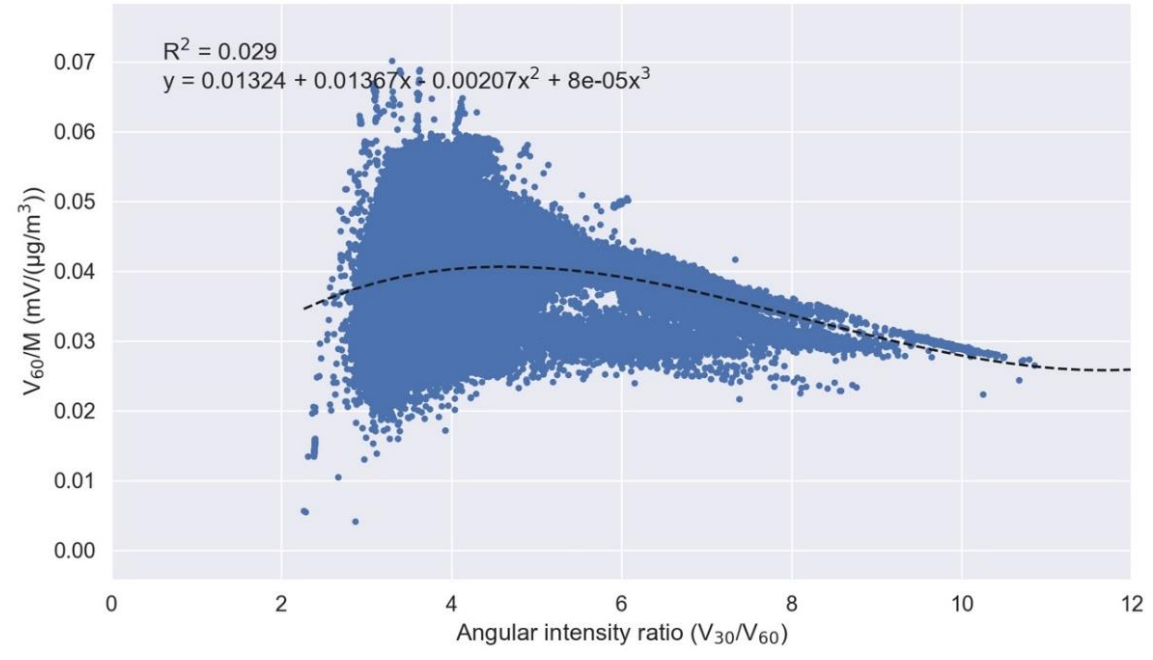

Fig. 6. Average mass sensitivity at $60^{\circ}$ as a function of the angular intensity ratio.

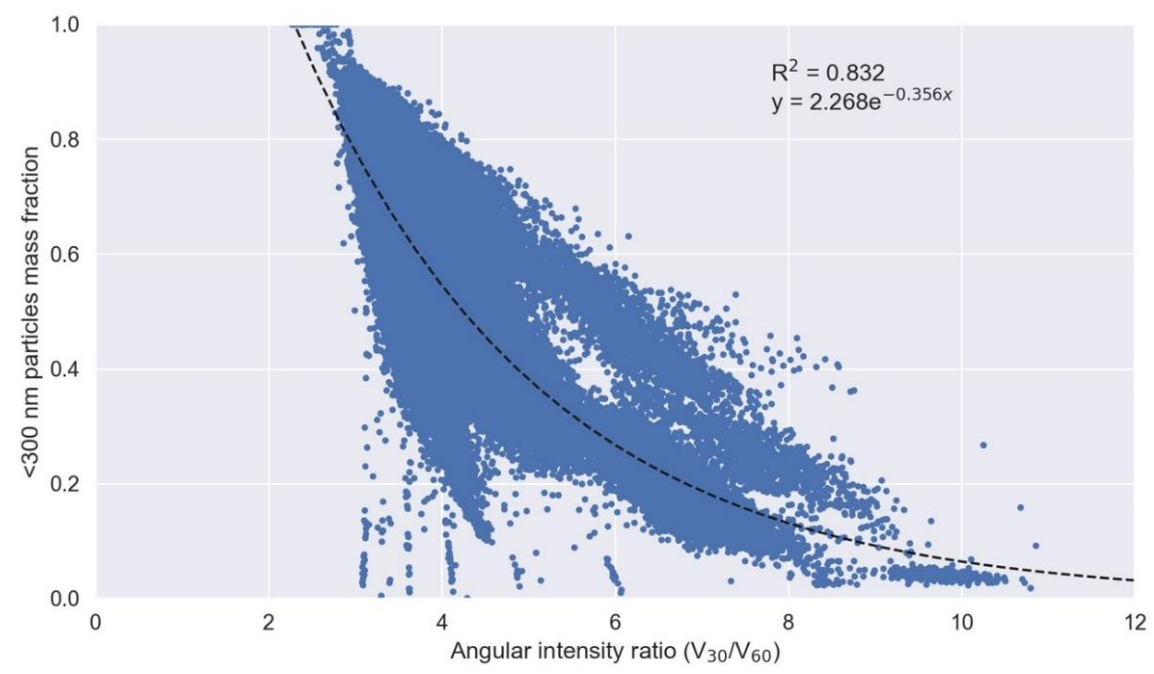

Fig. 7. Mass fraction of $\mathrm{PM}_{0.30}$ particles (defined as the fraction of total mass due to particles with diameters less than $300 \mathrm{~nm}$ ).

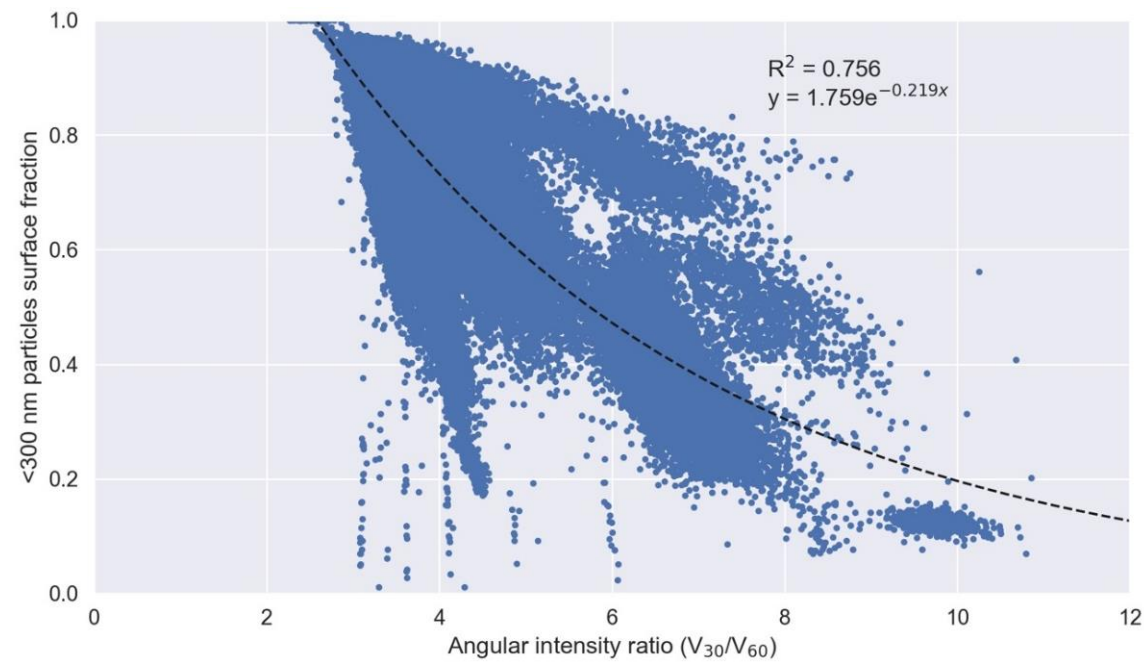

Fig. 8. Surface fraction of $P M_{0.30}$ particles (defined as the fraction of total particle surface due to particles with diameters less than $300 \mathrm{~nm}$ ). 


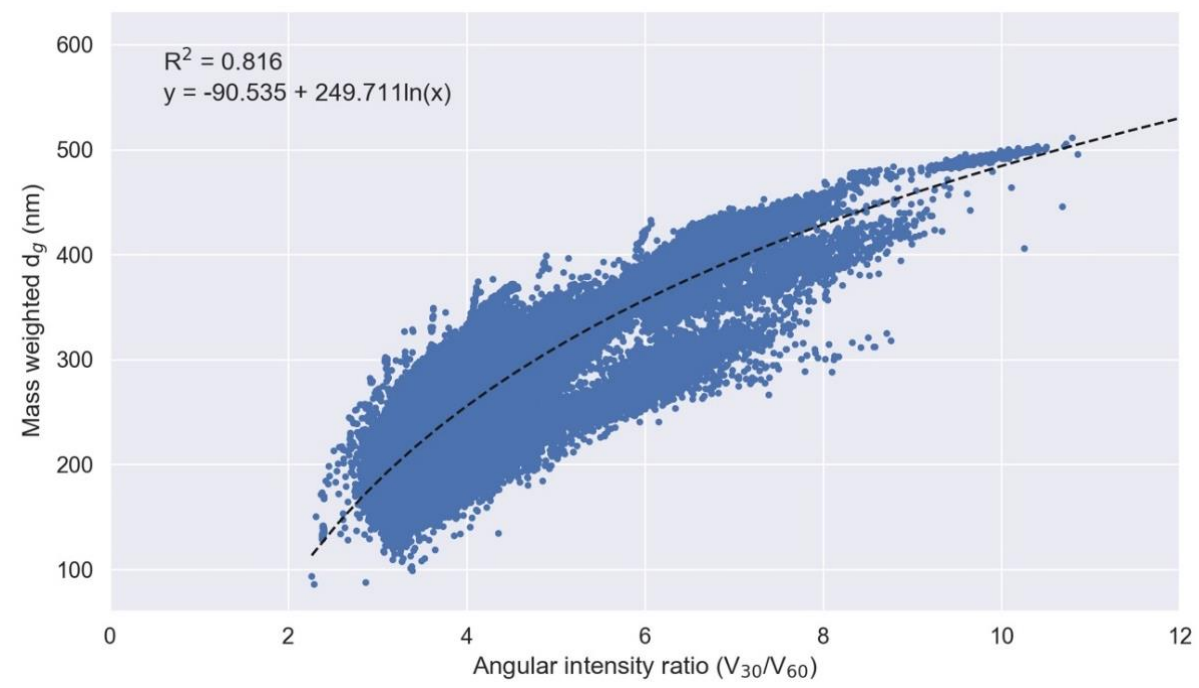

Fig. 9. Mass weighted average diameters as a function of the angular ratio.

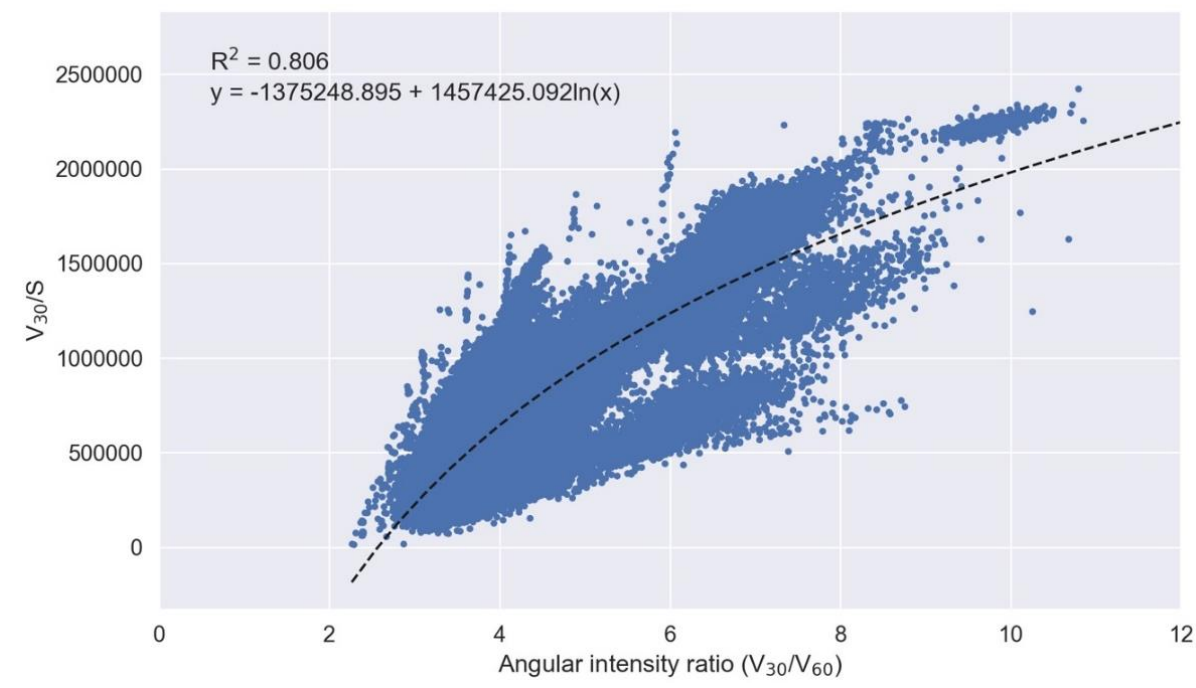

Fig. 10. Average surface sensitivity at $30^{\circ}$ as a function of the angular intensity ratio.

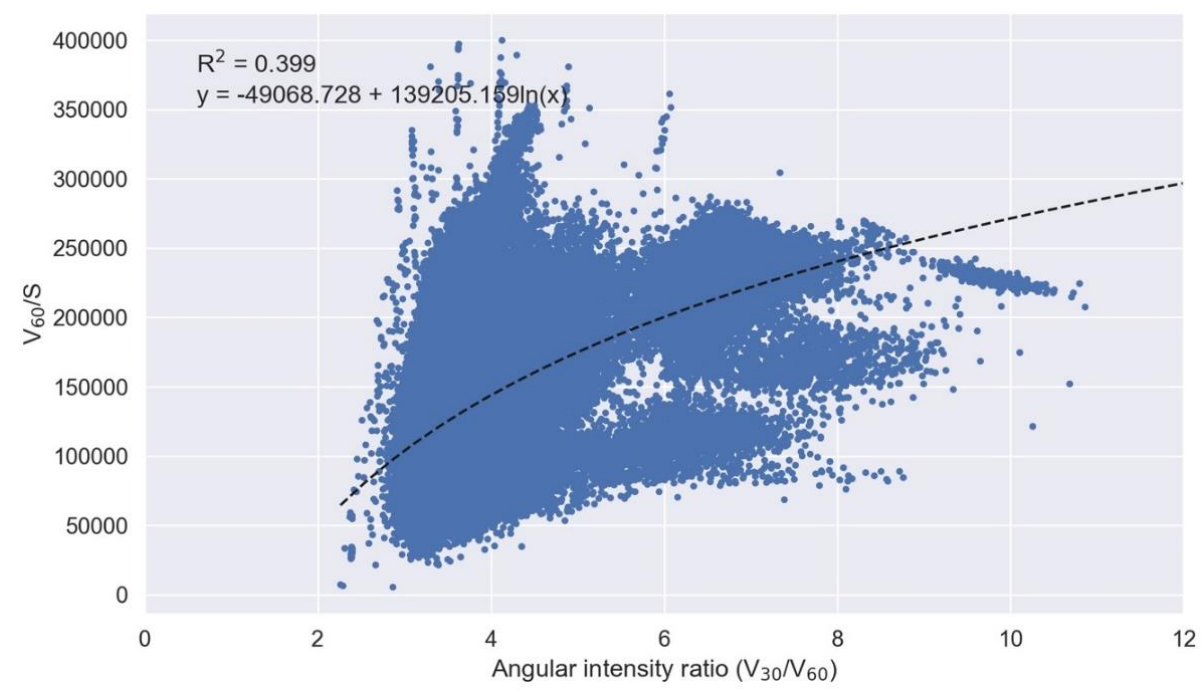

Fig. 11. Average surface sensitivity at $60^{\circ}$ as a function of the angular intensity ratio. 
Once the total mass concentration is known, the fraction of this mass that is due to particles with number weighted diameters less than $300 \mathrm{~nm}, \mathrm{PM}_{0.30}$ (Fig. 7) would then be determined from the measured voltage ratio. Multiplying this fraction by the total mass will yield the mass concentration of particles with diameters less than $300 \mathrm{~nm}$. A similar process would be used for determining total particle surface areas and the surface area of particles with diameters less than $300 \mathrm{~nm}$ using the regression expressions in Figs. 8, 10 and 11.

One particular episode relevant to these simulations serves to demonstrate the sensitivity of this technique when, during an approximate one-week monitoring period at the North Kensington site, the angular intensity ratio increased by a factor of 2 as shown in Fig. 12. Subsequent analysis of the data indicated that although the total mass concentrations did not change dramatically during this episode, the mass fraction of ultrafine particles showed a substantial decrease as evidenced in Fig. 13, decreasing from an average mass fraction of around $65 \%$ to a value of about $5 \%$ during this period, indicating a large increase in average particle diameters.

Consequently, the abrupt change in intensity ratio signaled a change in the mass distribution

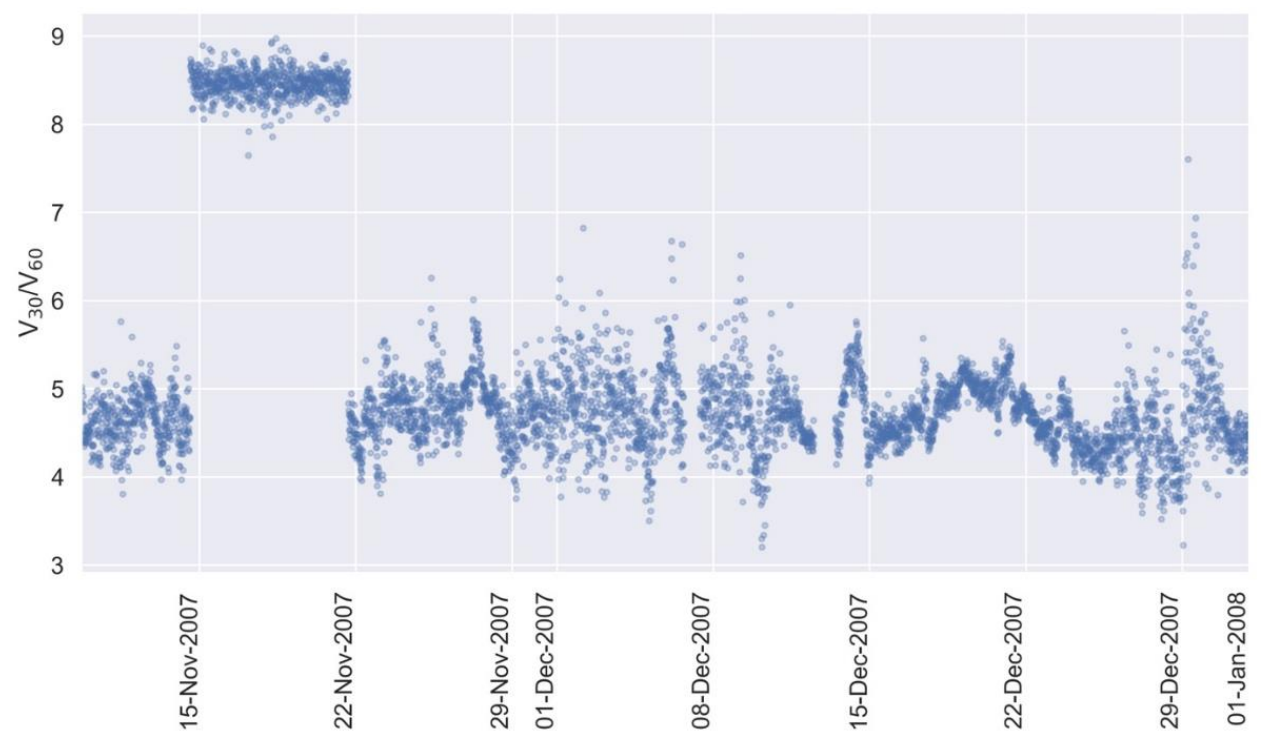

Fig. 12. Abrupt change in angular intensity ratio at the North Kensington site during November 2007.

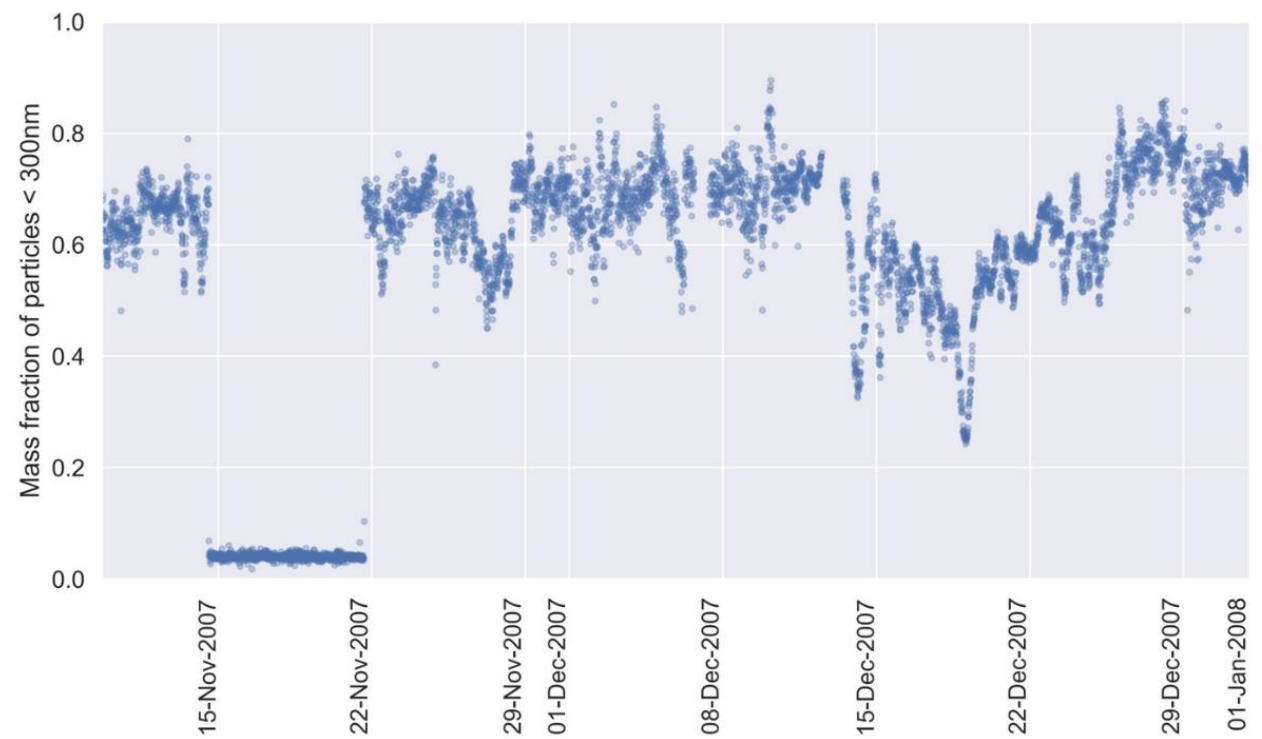

Fig. 13. Change in $\mathrm{PM}_{0.30}$ mass fraction corresponding to increase in angular intensity ratio at one of the monitoring locations. 
that the simulations readily revealed. This is the type of behavior one should expect during normal use of the proposed sensor.

\section{CONCLUSIONS}

The ratio of angular intensities serves a two-fold purpose in the final device. This ratio can be used first to define two sensitivity parameters that are capable of yielding a much-improved determination of the total particulate mass concentration and once this determination has been made this same ratio value is used to determine the fraction of that total mass that is due to very tiny particles with diameters less than $300 \mathrm{~nm}$ and the fraction that is due to larger particles.

While producing aerosols from various potential indoor (and outdoor) sources is definitely something that could be done in a laboratory setting for defining characteristic sensor responses to different particulate sources, it is doubtful that such experiments would be able to simulate the complex mix of particles from different sources (both indoors and outdoors) that might be typical of indoor environments. A better solution would be the use of these sensors alongside a continuously recording SMPS and possibly total mass monitor, such as a Tapered Element Oscillating Microbalance (TEOM), so that size, number, and mass data may be acquired while simultaneously measuring the angular intensities and their ratios from the same sample of airborne particles. These data would allow for a much-improved algorithm to decode the measured intensities and provide for a correspondingly more reliable determination of total particulate mass concentrations and ultrafine mass concentrations.

However, the correlations developed here should be sufficiently accurate to begin the application of this technique and to use the results to compare to reference instrumentation for further analysis and refinement.

\section{REFERENCES}

Barber, P.W., Hill, S.C. (1990). Light scattering by particles: Computational methods (Vol. 2). WORLD SCIENTIFIC. https://doi.org/10.1142/0784

Bell, M.L., Ebisu, K. (2012). Environmental inequality in exposures to airborne particulate matter components in the United States. Environ. Health Perspect. 120, 1699-1704. https://doi.org/ 10.1289/ehp.1205201

Department for Environment Food \& Rural Affairs (DEFRA) (2019). UK Particle Monitoring Programme. Retrieved December 7, 2019. https://uk-air.defra.gov.uk/data/particle-data

Dick, W.D., Ziemann, P.J., McMurry, P.H. (2007). Multiangle light-scattering measurements of refractive index of submicron atmospheric particles. Aerosol Sci. Technol. 41, 549-569. https://doi.org/10.1080/02786820701272012

Dietrich, J., Yermakov, M., Reponen, T., Kulkarni, P., Qi, C., Grinshpun, S.A. (2015). Protection of firefighters against combustion aerosol particles: Simulated workplace protection factor of a half-mask respirator (pilot study). J. Occup. Environ. Hyg. 12, 415-420. https://doi.org/10.108 0/15459624.2015.1006637

Geiss, O., Bianchi, I., Barrero-Moreno, J. (2016). Lung-deposited surface area concentration measurements in selected occupational and non-occupational environments. J. Aerosol Sci. 96, 24-37. https://doi.org/10.1016/j.jaerosci.2016.02.007

Hawley, B., McKenna, D., Marchese, A., Volckens, J. (2014). Time course of bronchial cell inflammation following exposure to diesel particulate matter using a modified EAVES. Toxicol. Vitr. 28, 829-837. https://doi.org/10.1016/j.tiv.2014.03.001

Health Effects Institute (HEI) (2013). Understanding the Health Effects of Ambient Ultrafine Particles: Perspectives 3. https://www.healtheffects.org/publication/understanding-healtheffects-ambient-ultrafine-particles

Kaye, P.H., Hirst, E. (2010). An improved low cost apparatus and method for the detection of a fluid borne-particle, UK Patent Application (Patent No. 2484930).

Kelly, F.J., Fussell, J.C. (2012). Size, source and chemical composition as determinants of toxicity attributable to ambient particulate matter. Atmos. Environ. 60, 504-526. https://doi.org/10.1 016/j.atmosenv.2012.06.039 
Kuuluvainen, H., Rönkkö, T., Järvinen, A., Saari, S., Karjalainen, P., Lähde, T., Pirjola, L., Niemi, J. V., Hillamo, R., Keskinen, J. (2016). Lung deposited surface area size distributions of particulate matter in different urban areas. Atmos. Environ. 136, 105-113. https://doi.org/10.1016/j.atm osenv.2016.04.019

Li, Z., Konisky, D.M., Zirogiannis, N. (2019). Racial, ethnic, and income disparities in air pollution: A study of excess emissions in Texas. PLoS One, 14, e0220696. https://doi.org/10.1371/journa I.pone.0220696

Litton, C.D., Cauda, E. (2019). Concerns about the use of inexpensive dust sensors to assess the air quality in urban environments. Submitt. to Journal, Inhal. Toxicol. (Awaiting Rev.)

Maynard, A.D., Kuempel, E.D. (2005). Airborne nanostructured particles and occupational health. J. Nanoparticle Res. 7, 587-614. https://doi.org/10.1007/s11051-005-6770-9

Morawska, L., Thai, P.K., Liu, X., Asumadu-Sakyi, A., Ayoko, G., Bartonova, A., Bedini, A., Chai, F., Christensen, B., Dunbabin, M., Gao, J., Hagler, G.S.W., Jayaratne, R., Kumar, P., Lau, A.K.H., Louie, P.K.K., Mazaheri, M., Ning, Z., Motta, N., Mullins, B., ... Williams, R. (2018). Applications of low-cost sensing technologies for air quality monitoring and exposure assessment: How far have they gone? Environ. Int. 116, 286-299. https://doi.org/10.1016/j.envint.2018.04.018

National Center for Biotechnology Information (2020). PubChem Compound Summary for CID 8346, Dioctyl phthalate. https://pubchem.ncbi.nlm.nih.gov/compound/Dioctyl-phthalate

Shi, L., Zanobetti, A., Kloog, I., Coull, B.A., Koutrakis, P., Melly, S.J., Schwartz, J.D. (2016). Lowconcentration $\mathrm{PM}_{2.5}$ and mortality: Estimating acute and chronic effects in a population-based study. Environ. Health Perspect. 124, 46-52. https://doi.org/10.1289/ehp.1409111

U.S. EPA (2008). Final Report | Ultrafine Particles: Characterization, Health Effects and Pathophysiological Mechanisms. https://cfpub.epa.gov/ncer_abstracts/index.cfm/fuseaction/ display.highlight/abstract/1098/report/F\#main-content

Unger, R.L. (1999). Particle sensor and related method offering improved particle discrimination, United States Patent (Patent No. 5,870,190).

Yale-NUS College Singapore and Yale University's School of Forestry and Environmental Studies. (2019). Urban Environment \& Social Inclusion Index Home I Data-Driven EnviroLab. https://datadrivenlab.org/urban/ (accessed 8 December 2019). 